\title{
IMPORTANCIA DE LA COMUNICACIÓN, RELACIONES PÚBLICAS Y TIC'S EN LAS ORGANIZACIONES DEL SIGLO XXI
}

Lcda. María José Menéndez Ledesma Docente titular tiempo completo Instituto Superior Tecnológico de Formación maria.menendez1@formacion.edu.ec

Palabras claves: organizaciones, comunicación, relaciones públicas, TIC'S.

Recibido: 7 de julio de 2016

Keywords: organizations, communication, public relations, TIC'S

Aceptado: 29 de noviembre de 2016

\section{RESUMEN}

El presente trabajo investigativo aborda de forma general el tema de la comunicación en las organizaciones y el vínculo que existe entre las TIC'S y las relaciones públicas debido a la creciente competitividad que existe actualmente en el mercado, viéndose reflejada en la similitud de productos y/o servicios existentes, motivo por el cual los empresarios se han visto en la necesidad de incorporar a las relaciones públicas como herramienta necesaria que pueda lograr diferenciar a la organización de sus competidores, con el fin de posicionar a la empresa en el mercado. En consonancia con ello, tanto las comunicaciones como la utilización de las relaciones públicas resultan ser fundamentales para construir y fomentar la imagen de una empresa, así como también su posicionamiento institucional, para lo cual se debe mantener buenas relaciones y una comunicación por el canal adecuado surgiendo con ello el creciente papel de las TIC'S que son consideradas hoy dia como una herramienta vital para el éxito de las empresas debido a su forma de aplicación y uso en las personas.

\section{ABSTRACT}

This research work generally addresses the issue of communication in organizations and the link between TIC's and public relations due to the increasing competition that exists in the market, reflected in the similarity of goods and / or existing services, why entrepreneurs have seen the need to incorporate public relations as a necessary tool that can achieve differentiate the organization from its competitors, in order to position the company in the market. In line with this, both communications and the use of public relations prove to be critical to build and promote the image of a company as well as its institutional position, for which it must maintain good relations and communication through the appropriate channel emerging thereby increasing role of TIC's that are considered today as a vital tool for business success due to its form of application and use in people. 


\section{INTRODUCCIÓN}

Con el paso del tiempo, la tecnología ha ido evolucionando vertiginosamente. Hoy día el rol protagónico lo tiene el internet con sus múltiples aplicaciones, lo que ha significado una verdadera revolución para la sociedad y enorme beneficio para las empresas, debido a la eficaz y rápida transmisión de información y comunicación que realizan por medio de las redes sociales, actualmente, uno de los canales más utilizados por las organizaciones para dar a conocer los productos.

Una evidencia de lo antes expuesto la aporta la telefonía celular que en los últimos 20 años ha ido creciendo cada vez más rápido, sobre todo en los países en desarrollo.Así al ser América Latina parte del mundo en desarrollo, naciones como Argentina, Brasil, Chile, México, Perú, Ecuador y Venezuela cuentan con un alto crecimiento en utilización de tecnologías de información y comunicación.

Claramente las TIC'S han llevado a América Latina a nuevas e innovadoras actividades y es por ello que la mayor parte de las empresas de hoy en día no conciben ni asumen su desarrollo sin la aplicación de las Tecnologías de la Información y Comunicación.

Asimismo las Relaciones Públicas se han convertido en una herramienta importante para las organizaciones debido a su carácter innovador y creativo que dan acceso a nuevas formas de comunicación, sirviendo, de esta manera, de apoyo a los empresarios para presentar y vender sus productos o servicios a través del internet con menores costos.

Sin embargo se ha identificado una problemática social en América Latina, respecto al efecto de las TIC'S en la comunicación humana debido a que no está siendo bien utilizada por algunas empresas y por la mayor parte de las personas, especialmente los jóvenes convirtiéndose en una arma negativa para el desarrollo tecnológico de un país.

En este sentido, el objetivo del presente artículo consiste en realizar una revisión documental con el fin de analizar el efecto y la importancia de la comunicación, las relaciones públicas y las TIC'S en las organizaciones del siglo XXI, con el fin de analizar en qué medida la tecnología ha incidido positiva y negativamente en las organizaciones y como pueden las estructura basadas en las relaciones públicas y las TIC'S construirse en herramientas estratégicas para aprovechar las oportunidades del mercado ya que se vive a diario una alta competencia entre las empresas.

El contenido de este artículo se organiza en cuatro secciones diferentes. En la primera se da a conocer la importancia de la comunicación para las organizaciones del siglo XXI, dentro de ella se citan algunos de los aspectos favorables de las Relaciones Públicas, la implementación de las TIC'S en las empresas, así como la situación actual de la sociedad en cuanto al uso de la tecnología.

En la segunda sección se analiza la recomendación de la buena implementación de las TIC'S en las organizaciones. En la tercera parte se estudia como las relaciones públicas y las TIC'S pueden ser manejadas estratégicamente en beneficio de las empresas y finalmente se establece las conclusiones del trabajo 


\section{DESARROLLO}

\section{Importancia de la comunicación, relaciones públicas y Tic's en las organizaciones del siglo XXI}

La comunicación ha ido desempeñando un papel de suma importancia en la vida cotidiana del ser humano, debido a que el hombre siempre se ha visto en la necesidad de comunicarse con otras personas con la finalidad de transmitir o recibir significados a través de un sistema compartido de signos y normas semánticas.

Martínez \& Nosnik (1988) mencionan que la comunicación "es un proceso por medio del cual una persona se pone en contacto con otra a través de un mensaje, y espera que está última de una respuesta".

Puede ser considerado por muchos como un proceso simple pero a la vez es muy complejo e importante para la vida humana, que involucra elementos conocidos como lo son: emisor, mensaje, canal, receptor y la retroalimentación el cual se lleva a cabo entre dos o más personas. Sin una correcta comunicación por parte de las empresas, es imposible que puedan lograr metas, aumentar productividad y generar los resultados que se deben alcanzar.

Actualmente con la evolución tecnológica, las empresas han identificado la importancia y necesidad de comunicar por nuevos canales con la finalidad de hacer llegar un mensaje determinado, encontrándose la participación de las Tecnologías de la Información y la Comunicación, también conocida como TIC, que son el conjunto de tecnologías desarrolladas para gestionar información y enviarla de un lugar a otro. Abarcan un abanico de soluciones muy amplio. Incluyen las tecnologías para almacenar información y recuperarla después, enviar y recibir información de un sitio a otro, o procesar información para poder calcular resultados y elaborar informes. (Sevicios TIC)

Para Escalante (2013), las TIC'S son una conjunción de tres elementos fundamentales, en primer lugar las telecomunicaciones, el área de la informática y la menos visible, la micro-electrónica, las cuales abarcan todos los ámbitos de la sociedad, cambiando y afectando directamente nuestro entorno y las capacidades del proceso de comunicación entre las personas, las máquinas y la integración de ambos.

Con relación a las TIC'S, para Cabero (2001), se ha concebido como un fenómeno que toca a la sociedad en todas sus dimensiones y rebasa aspectos específicamente técnicos y tecnológicos, pues es un acontecimiento con implicaciones en los niveles político, cultural, económico y social.

Dentro de las principales características de estas tecnologías Cabero (1996) destaca las siguientes:

- La inmaterialidad entendida desde una doble perspectiva: la consideración de que la materia prima es la información y la posibilidad de crear mensajes sin la existencia de un referente externo.

- La instantaneidad hace referencia a la rapidez en el acceso a la información, haciendo una ruptura de las barreras temporales y espaciales de naciones y culturas. 
- La posesión de altos niveles de calidad y fiabilidad debido a la digitalización de la información.

- La facilidad de manipulación y distribución de la información.

- Las altas posibilidades de interconectarse.

- La interactividad.

- La innovación en cuanto que las nuevas tecnologías persiguen como objetivo la mejora, el cambio y la superación cualitativa y cuantitativa de las tecnologías anteriores.

En el caso específicamente de Ecuador, de acuerdo a la última encuesta realizada por el Instituto Nacional de Estadísticas y Censo en el mes de Diciembre del 2015 indica que: el $36,88 \%$ de las empresas realizaron innovación en procesos, encasillándose a las empresas de prestaciones de servicios con un 44,3\%, lo cual refleja la utilización de las TIC'S. ( Figura 1 y 2 )

\section{Figura 1}

Porcentaje de empresas según tipo de innovación.

\section{PORCENTAJE DE EMPRESAS SEGÚN TIPO DE INNOVACIÓN}

El $36,21 \%$ de las empresas ecuatorianas introdujeron innovaciones de productos

Innovación en proceso

Innovación de producto

Innovación comercialización

Innovación organizacional

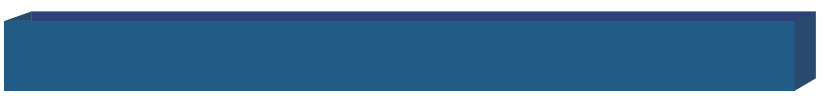

$36,88 \%$

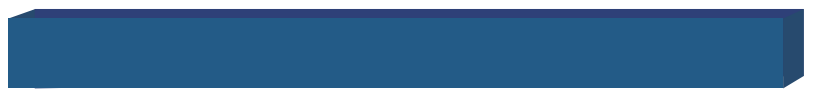

$36,21 \%$

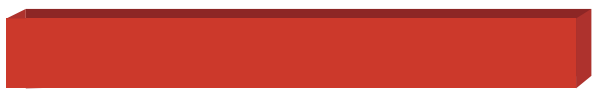

$21,35 \%$

$20,94 \%$ 


\section{Figura 2}

Tipo de innovación desagregado por actividad económica.

\section{TIPO DE INNOVACIÓN DESAGREGADO POR ACTIVIDAD ECONÓMICA}

El sector de Servicios es la actividad económica más propensa e innovadora en el país

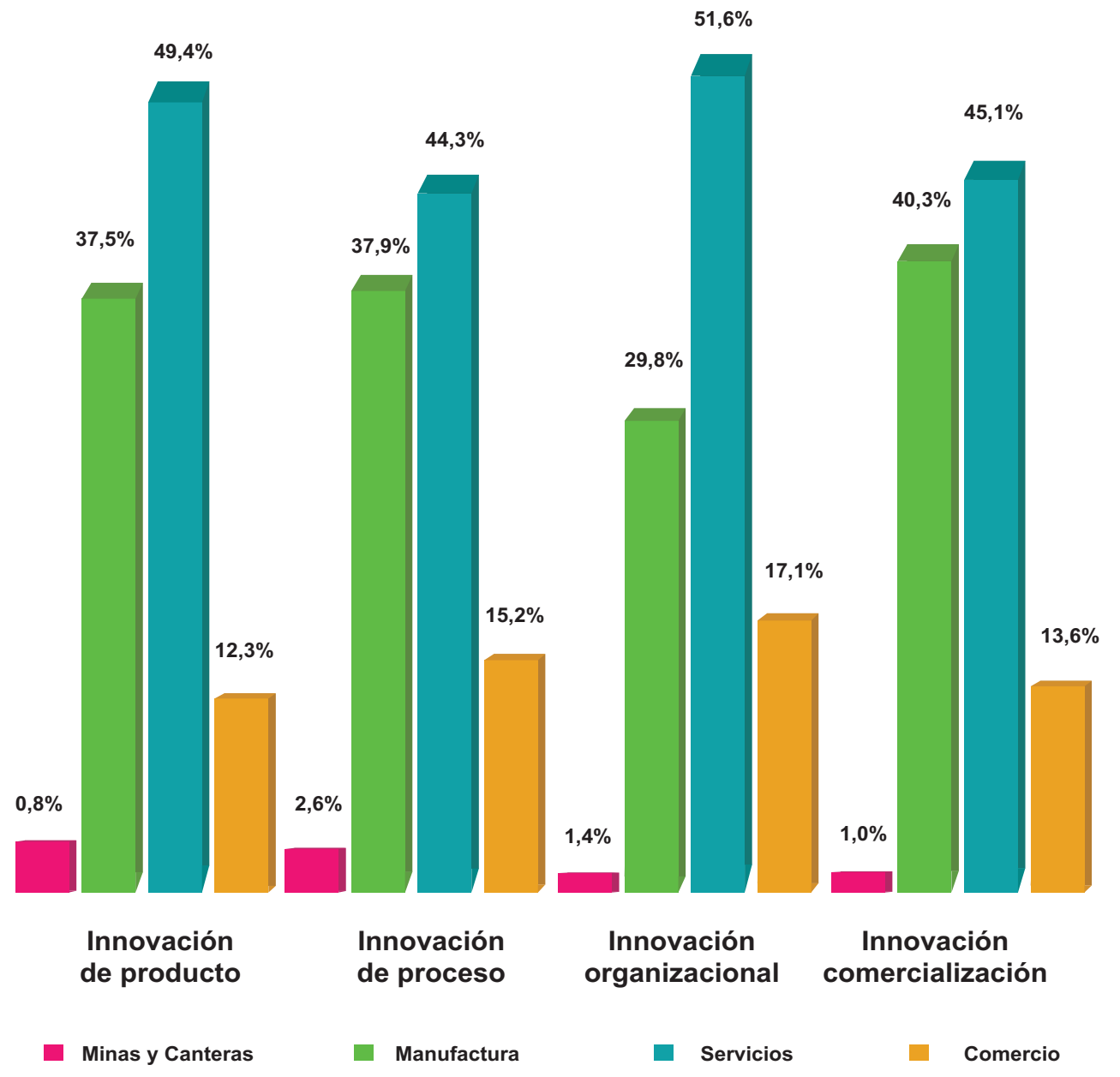

Fuente: (Tecnologías de la Información y Comunicaciones TIC'S , 2015)

Asimismo se cuenta con el $89,90 \%$ de población que utiliza celulares y redes sociales encontrándose en una edad comprendida desde los 5 años en adelante. (Figura 3) 


\section{Figura 3}

Porcentaje de población con celulares y redes sociales.

\section{PORCENTAJE DE POBLACIÓN CON CELULARES Y REDES SOCIALES}

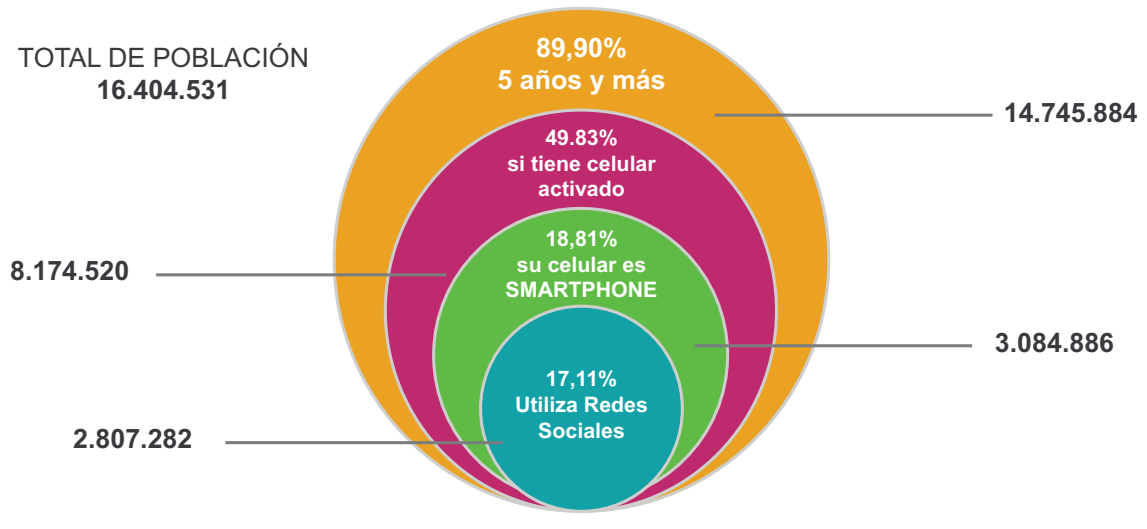

Fuente: (Tecnologías de la Información y Comunicaciones TIC'S , 2015)

Se evidencia como la tasa de crecimiento per cápita del número de usuarios de Internet han sido mayores en los países en desarrollo que en los desarrollados.

Es válido señalar que para que la telefonía convencional estuviera al alcance de la mayoría debió transcurrir alrededor de 100 años y en el caso de la televisión 50 años, sin embargo para las TIC'S 15 años han sido suficiente.

Notoriamente las TIC's han ofrecido nuevas e innovadoras posibilidades en el contexto latinoamericano. De acuerdo a un estudio realizado por la Comisión Económica para América Latina y el Caribe, el CAF Banco de Desarrollo de América Latina, el CET.LAT Centro de Estudios de Telecomunicaciones de América Latina y la Fundación Telefónica, se han identificado las transformaciones de la industria de las telecomunicaciones y de servicios basados en el internet, donde señalan que, en la última década en Latinoamérica se ha logrado avances muy relevantes en la adopción de tecnología digitales. Entre el año 2006 y 2013 se ha multiplicado el uso de usuarios en el internet, principalmente por la caída de los precios de la banda ancha. En la actualidad el $47 \%$ de la población es internauta, lo que significa que son personas que utilizan los servicios de internet u otra red informática. (El ecosistema y economía digital de América Latina, 2015)

Es por ello que se puede encontrar actualmente en las empresas desde un equipamiento básico de las TIC'S como lo es la conexión a internet, hasta la disposición de banda ancha para las comunicaciones móviles, asimismo algunas organizaciones facilitan a sus empleados equipos móviles como laptops y celulares con el objetivo que se mantengan comunicados con los clientes.

Por tal motivo, gracias a las TIC'S, las organizaciones han logrado alcanzar importantes beneficios, entre ellos: apertura de nuevos mercados, nuevos modelos de negocio, 
comunicación con los clientes, automatización de los procesos de compra, ahorro de costes, agilidad en la toma de decisiones, facilidad en la comunicación tanto para los clientes internos como externos y un mayor conocimiento acerca de las necesidades de los clientes.

De esta manera las TIC'S se aplican a todas las áreas de una empresa tanto Financiera, Marketing, Ventas, Recursos Humanos, Producción, Logística y Distribución, desarrollando cada vez más herramientas específicas para cada área, como por ejemplo: los sistemas de planificación de recursos empresariales que se encargan de la facturación y el manejo del proceso interno, así mismo el comercio electrónico encontrando a (B2B) Business to Business,(B2C) Business to Customer y (B2G) Business to Gobierno; sistemas de colaboración y trabajo en equipo como correo electrónico, redes sociales con la finalidad de trabajar en equipo de forma simultáneamente; CRM Customer Relationship Management que permiten desarrollar vínculos con los clientes; plataformas y redes sociales; portales empresariales y blogging lo cual se ha convertido en la última tendencia para las empresas.

Por tal razón las organizaciones del siglo XXI están inmersas en una sociedad que hace cada día más utiliza las Tics en su diario quehacer, evidenciado por el uso de las tablets, teléfonos inteligentes, consolas de juego con realidad virtual, cámaras fotográficas, barreras de acceso, wifi en todos lados, cajeros automáticos, cámaras de video, entre otros dispositivos electrónicos inteligentes, lo que hace importante que los gerentes estén en condiciones de ver la realidad y estar a la vanguardia para generar mayor economía en las empresas, de otra manera se perdería mercado.

Sin embargo, a nivel mundial y como consecuencia de la globalización, en América Latina la economía se ha caracterizado no solo por los avances tecnológicos sino también por el desarrollo de los productos. Es así como las empresas del siglo XXI crean cada vez más productos nuevos e innovadores cuyo fin es llegar a su público objetivo, satisfacer las necesidades de los consumidores y ganar posicionamiento en el mercado, lo que obliga a las empresas a comprender mejor estos paradigmas comerciales con la finalidad de poder crear estrategias y lograr ser exitosas tanto en el mercado local donde se desarrollan como a nivel de otros países.

Es por ello que en la actualidad las Relaciones Públicas se han convertido en una herramienta fundamental e indispensable para las empresas, sean pequeñas, medianas o grandes, pues se convierte en la herramienta que se encarga de lograr con beneficio la satisfacción del consumidor por medio de la comunicación. Por ello, toda empresa requiere de la implementación del área de Relaciones Públicas para ser exitosa en el mercado, debido a que es la que se encarga de ayudar en las organizaciones en todas y cada una de las actividades que realizan, encontrándose entre ellas a: consultoría en estrategias y campañas de comunicación, estudios de opinión, auditoria de comunicación, relaciones con los medios de comunicación, gestión de entrevistas, seguimiento de medios, comunicación interna, comunicación integral de marketing, relaciones corporativas, comunicación electrónica, realización de eventos, entre muchas más actividades.

Por tal motivo el concepto de relaciones públicas encierra muchos temas y se relaciona con diversas áreas, en este artículo se destaca una de las primeras definiciones, internacionalmente aceptada, presentada por la Fundación para la Educación e Investigación de las Relaciones Públicas. 
En el año 1975 Rex Harlow estudió 472 propuestas de definiciones y en un intento por reflejar la esencia de sus principales contenidos, señaló lo siguiente:

Las relaciones públicas son una función directiva específica que ayuda a establecer y a mantener líneas de comunicación, comprensión, aceptación y cooperación mutuas entre una organización y sus públicos; implica la resolución de problemas y cuestiones; define y destaca la responsabilidad de los directivos para servir al interés general; ayuda a la dirección a mantenerse al tanto de los cambios y a utilizarlos eficazmente sirviendo como un sistema de alerta inmediata para ayudar a anticipar tendencias; y utiliza la investigación y las técnicas de comunicación éticas y sensatas como herramientas principales (Rex, 1976)

Por otra parte José Luis Arceo Vacas indica en su libro "Fundamentos para la teoría y la técnica de las Relaciones Públicas" que las Relaciones Públicas tienen su fundamento en la comunicación". (Vacas, 1988)

De la misma manera Sam Black, profesor honorario de Relaciones Públicas de la Universidad de Stirling y una de las voces más autorizadas del mundo en esta rama, quien fue presidente de International Public Relations Association IPRA, indica: "La práctica de las Relaciones Públicas es el arte y la ciencia de conseguir la armonía con el entorno por medio de la compresión mutua, basada en la verdad y en una información completa"

Por consiguiente, las organizaciones hoy en día requieren de profesionales de relaciones públicas con conocimientos adecuados a su carrera, debido a que son los encargados de realizar una gran cantidad de actividades específicas que ayuden a las organizaciones a comunicarse exitosamente con todas sus audiencias con la finalidad de mantener e incrementar la satisfacción de los clientes y la participación del mercado.

Durante los últimos años se ha podido presenciar cómo las relaciones públicas han ido cambiando radicalmente gracias a la tecnología, convirtiéndose en una herramienta de fácil acceso al Internet, apareciendo de esta manera el comercio electrónico, las TIC y los contenidos digitales que están marcando las tendencias en el Marketing Digital evidenciándose en el escenario de la comercialización de servicios y productos de las empresas tanto a nivel local, nacional y mundial.

Por tal motivo se puede concluir que el creciente desarrollo tecnológico ha ido penetrando y transformado la forma de cómo los seres humanos se comunican, piensan y hasta ha cambiado el comportamiento de compra, apareciendo una nueva era denominada "era del conocimiento", que tiene a las Tecnologías de la Información y la Comunicación TIC's y las Relaciones Públicas como una de las influencias de mayor impacto en nuestro tiempo.

\section{CONCLUSIONES}

La comunicación, las TIC'S y Relaciones Púbicas en las organizaciones del siglo XXI, son elementos fundamentales pues constituyen parte esencial para el éxito empresarial como consecuencia de la creciente similitud de productos y servicios que existen en el mercado.

Sobre la base de la investigación realizada, se resalta que es importante que toda pequeña y mediana empresa recurra a un servicio de Relaciones Públicas para anunciar asuntos relevantes al lanzamiento de nuevos productos o servicios, apertura de un nuevo 
establecimiento, difusión de próximos eventos, promoción de web sites, introducción de nuevos productos o servicios, realizar campañas de concientización que despierten el interés de la opinión pública y que le brinden una excelente imagen institucional, entre otros. Por tal motivo es importante que en toda organización exista un departamento interno de comunicación y relaciones públicas, para que tenga permanente contacto con el mercado, y pueda manejar una buena comunicación de los productos y/o servicios que ofrecen para los clientes.

Otro factor que debe considerarse, es que las TIC'S son claves para el desarrollo de nuevos productos y nuevos modelos de negocios de las empresas, de la misma forma permite tener una mayor cercanía con los clientes y proveedores, también ayudan en la sistematización de los procesos internos de las organizaciones con la finalidad de alcanzar la excelencia operacional, produciendo más en menor tiempo.

Si bien es cierto, hoy en día la mayor parte de las personas utilizan el internet, por tal motivo las empresas se ven en la necesidad de buscar herramientas eficaces e idóneas que lleguen a su grupo objetivo con la finalidad en dar a conocer los beneficios, bondades, características de un producto o servicio determinado.

En secuencia con la influencia del Internet en la sociedad, existen nuevos lenguajes y formas de realizar algunas actividades, entre ellas se cuenta con el envío de mensajes ya no de textos sino por medio de aplicaciones como lo son: Messenger, whatsapp, video llamadas, lo cual ha modificado de cierta forma el comportamiento y manera de pensarrazonar de las personas. 


\section{REFERENCIAS BIBLIOGRÁFICAS}

Rex, H. (1976). Building a public relations definition $n^{\circ} 4$.

Sevicios TIC. (s.f.). http://www.serviciostic.com/las-tic/definicion-de-tic.html

Vacas, J. L. (1988). Fundamentos para la teoría y la técnica de las relaciones públicas.

ttp://blogs.eada.edu/2012/07/05/tecnologias-informacion-en-empresa

http://www.ecuadorencifras.gob.ec//documentos/web inec/Estadisticas_Economicas/Ciencia_Tecnologia/Presentacion_de_principales_resultados_ACTI.pdf

http://www.ecuadorencifras.gob.ec//documentos/web-inec/Estadisticas_Sociales/ TIC/2015/Presentacion_TIC_2015.pdf

https://www.youtube.com/watch?v=JUrhX3-aYhE.El ecosistema y economía digital de América Latina. (10 de Agosto de 2015). Recuperado el 5 de Octubre de 2016, de

http://negocios2015.blogspot.es/1447703783/las-importancia-de-las-tics-en-las-organizaciones/ 